\title{
A rare e14a3 BCR/ABL fusion transcript in acute lymphoblastic leukemia patient treated with CAR-modified T-cell therapy
}

\author{
HAODONG CAI, LI YANG, KEFENG SHEN, WEI ZHANG, JIE XIONG, \\ MEILAN ZHANG, XIA MAO, YING WANG and MIN XIAO \\ Department of Hematology, Tongji Hospital, Tongji Medical College, \\ Huazhong University of Science and Technology, Wuhan, Hubei 430030, P.R. China
}

Received August 8, 2017; Accepted November 21, 2017

DOI: $10.3892 / \mathrm{ol} .2017 .7611$

\begin{abstract}
E14a3 breakpoint cluster region (BCR)/ABL proto-oncogene 1, non-receptor tyrosine kinase (ABL) fusion transcript is rare in Philadelphia chromosome positive disease, particularly in acute lymphoblastic leukemia (ALL). Recently an e14a3 fusion transcript was detected by multiple laboratory examinations, and the patient was suffering from ALL. Except for the BCR/ABL fusion gene, in the present study the patient additionally had an IKAROS family zinc finger 1 deletion which, has been confirmed as a significant adverse prognosis factor. Following 2 rounds of chemotherapy, the patient presented cytological remission; however, the patient then relapsed 2 months later. They then received chimeric antigen receptor modified (CAR-modified) T-cell therapy and achieved complete remission. CAR-modified T-cell therapy is a powerful novel therapy which, exhibited great potential for treating refractory ALL, regardless of the existence and form of the BCR/ABL fusion transcript.
\end{abstract}

\section{Introduction}

Philadelphia $(\mathrm{Ph})$ chromosome is named for the city in which this special aberrant translocation of chromosome 9 and chromosome 22 was found. $\mathrm{Ph}$ chromosome is a key indication to diagnosis chronic myeloid leukemia (CML) and it is also found in approximately $20 \%$ acute lymphoblastic leukemia (ALL) patients and occasionally found in other hematological malignancies (1). $\mathrm{Ph}$ chromosome gives rise to a breakpoint cluster region $(\mathrm{BCR}) / \mathrm{ABL}$ proto-oncogene 1 , non-receptor tyrosine

Correspondence to: Dr Min Xiao, Department of Hematology, Tongji Hospital, Tongji Medical College, Huazhong University of Science and Technology, 1095 Jiefang Avenue, Hankou, Wuhan, Hubei 430030, P.R. China

E-mail: xiaomin@tjh.tjmu.edu.cn

Key words: acute lymphoblastic leukemia, breakpoint cluster region/ABL proto-oncogene 1, non-receptor tyrosine kinase, CAR-modified T-cell Therapy, e14a3 (b3a3), Philadelphia chromosome, imatinib therapy kinase $(\mathrm{ABL})$ fusion gene that results to the constitutive activation of proto-oncogene ABL1 and leads to oncogenesis. A variety of $\mathrm{BCR} / \mathrm{ABL}$ transcript variants have been reported such as e13a2 (b2a2), e14a2 (b3a2) which account for more than $95 \%$ in CML while ela2 accounts for more than $50 \%$ in ALL (2). Rare transcript variants are occasionally reported, including e8a2, e6a2, e14a3 etc. In terms of e14a3, most of the cases reported are males and diagnosed with $\mathrm{CML}$ or atypical CML. As to our knowledge, only 4 e14a3 (b3a3) BCR/ABL fusion transcript in ALL cases have been reported, with two children and two adults (3-6). Except for one lacking clinical data, all of them received chemotherapy and two received imatinib therapy additionally. Here we reported a case of e14a3 (b3a3) BCR/ABL fusion transcript in an adult ALL patient who have received chimeric antigen receptor modified (CAR-modified) T-cell therapy and this is the first report about CAR-modified T-cell therapy applied in a rare BCR/ABL fusion.

\section{Materials and methods}

Patient history. The present study was approved by the medical ethics committee of Tongji Hospital (Tongi Medical College, Huazhong University of Science and Technology, Hubei, China) and written informed consent was obtained from the patient. A 47-year-old male patient presented with low-grade fever and chest tightness. Hemoglobin was $65 \mathrm{~g} / 1$, leucocyte count was $299 \times 10^{9} / 1$, and platelet count was $45 \times 10^{9} / 1$. Bone marrow cytology showed that the white blood cell count was significantly increased that primitive and immature lymphocytes accounted for $87 \%$. Clonal immunoglobulin gene rearrangement detection showed a single clonal peak in detection range of IGHA and IGHB respectively. Cytogenetic analysis and fluorescent in situ hybridization (FISH) revealed a complex karyotype and a $\mathrm{Ph}$ chromosome respectively. Gene scanning and PCR found Ikaros6 transcription (Fig. 1A). After two rounds of chemotherapy he got a cytological remission but relapsed two months later. Then he received CAR-modified T-cell therapy that modified T cells targeting on the lineage-specific antigens CD19 were infused into body at the first time and followed with infusion of modified $\mathrm{T}$ cells targeting on CD22 for the next two times. Complete remission was soon achieved after the first CAR-modified T-cell therapy 
A Case sample

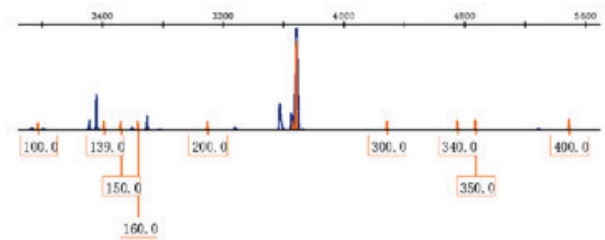

Agarose gel electrophoretogram

BV-173 Case Patient 1 Patient 2 Paticnt 3 Marker
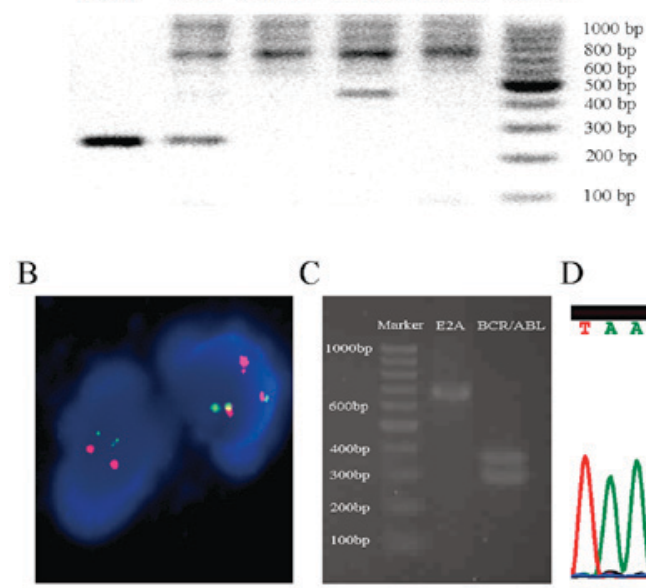

D
Positive control

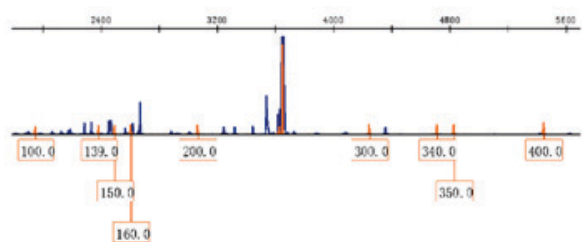

Negative control
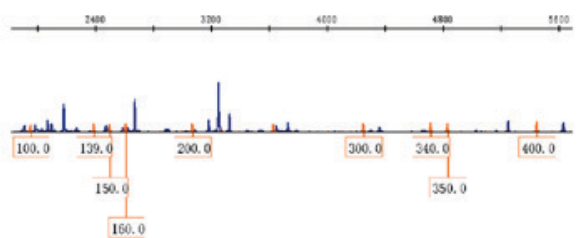

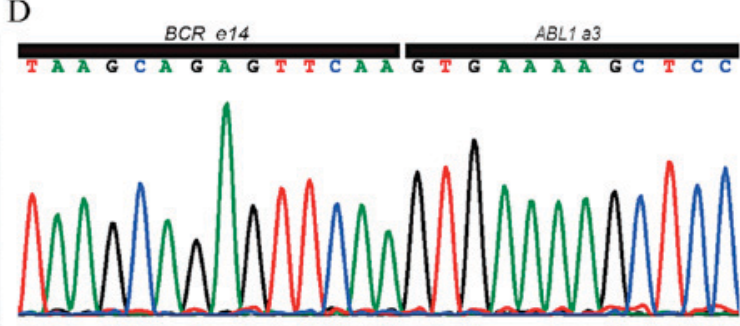

Figure 1. Summary of cytogenetic and molecular examinations. (A) Gene scanning and PCR with agarose gel electrophoresis detecting Ikaros6, revealed a positive result. In the agarose gel electrophoretogram, BV-173 served a role as the positive control as this cell line presented marked Ikaros6 transcription. (B) BCR/ABL fusion detected by fluorescent in situ hybridization. Normal cells presented a pattern of two green and two red signals, while cells with the BCR/ABL fusion gene exhibited yellow signal (fusion signal). (C) Two bands with one $\sim 300$ bp and another $\sim 380$ bp appeared in the agarose gel which, match the size of the BCR/ABL e14a3 fusion gene products in nested PCR. E2A works as an internal inference. (D) Sanger sequencing of the plasmid DNA with insertion of the fusion gene fragment. BCR exon 14 is joined with ABL1 exon 3 without extra sequence, gaining or losing.

Table I. Primer sequences and thermocycling conditions for nested polymerase chain reaction.

Thermocycling conditions

Primer sets

Primer sequence (5'-3')

First round

Second round

First round

BCR:3060U23

ABL:661L20

Second round

BCR:3128U22

ABL:642L23

\section{GAGTCACTGCTGCTGCTTATGTC TTTTGGTTTGGGCTTCACAC}

\section{CACGTTCCTGATCTCCTCTGAC} ACACCATTCCCCATTGTGATTAT $95^{\circ} \mathrm{C}$ for $5 \mathrm{~min}$

$95^{\circ} \mathrm{C}$ for $30 \mathrm{sec}$

$58^{\circ} \mathrm{C}$ for $30 \mathrm{sec}$

$72^{\circ} \mathrm{C}$ for $1 \mathrm{~min}$

Held at $4^{\circ} \mathrm{C}$

1 cycle
25 cycles

1 cycle
25 cycles

$95^{\circ} \mathrm{C}$ for $5 \mathrm{~min}$

$95^{\circ} \mathrm{C}$ for $30 \mathrm{sec}$

$58^{\circ} \mathrm{C}$ for $30 \mathrm{sec}$

$72^{\circ} \mathrm{C}$ for $1 \mathrm{~min}$

$72^{\circ} \mathrm{C}$ for $10 \mathrm{~min}$

Held at $4^{\circ} \mathrm{C}$
1 cycle

20 cycles

1 cycle

Table II. Breakpoint cluster region/ABL proto-oncogene 1, non-receptor tyrosine kinase fusion transcript detected by reverse transcription-quantitative polymerase chain reaction during the clinical course.

\begin{tabular}{llcc}
\hline Clinical course & Material & Transcript detected & Quantitative result \\
\hline Initial diagnosis & Bone marrow & $\mathrm{e} 14 \mathrm{a} 3$ & $108.30 \%$ \\
One month after chemotherapy completed & Bone marrow & $\mathrm{e} 14 \mathrm{a} 3$ & $0.19 \%$ \\
Two months after chemotherapy completed & Bone marrow & $\mathrm{e} 14 \mathrm{a} 3$ & $2.44 \%$ \\
Three months after chemotherapy completed & Bone marrow & $\mathrm{e} 14 \mathrm{a} 3$ & $110.48 \%$ \\
Two months after the 1st CAR-T therapy & Bone marrow & $\mathrm{e} 14 \mathrm{a} 3$ & $0.01 \%$ \\
One month after the 2nd CAR-T therapy & Bone marrow & - & 0 \\
One month after the 3rd CAR-T therapy & Bone marrow & - & 0
\end{tabular}

CAR-T, CAR-modified T-cell. 
without obvious adverse events except for a short period of fever. Up to date, he has maintained a complete cytogenetic remission for 5 months.

Cytogenetic analysis and FISH. Cells from bone marrow were cultured for 24-48 $\mathrm{h}$ and performed with Giemsa staining and GTG-banding according to standard cytogenetic laboratory protocols. FISH probe (Beijing GP Medical Technologies, Beijing, China) was applied to detect BCR/ABL rearrangement following the protocol manufacturer recommended.

Gene scanning for detection of IKaros6. Primers were 5'-ATG GATGCT GATGAGGGTCAAGAC-3' forward and 5'-GATGGCTTGGTCCATCACGTGG-3' reverse. cDNA was reverse transcribed from total RNA which was extracted from bone marrow based on routine protocol of our laboratory. GeneScan ${ }^{\mathrm{TM}} 500 \mathrm{LIZ}^{\mathrm{TM}}$ dye Size Standard and $\mathrm{Hi}_{-} \mathrm{Di}^{\mathrm{TM}}$ Formamide (both from Applied Biosystems, America) were mixed with PCR product and loaded on AB 3500 Genetic Analyzer (Applied Biosystems; Thermo Fisher Scientific, Inc., Waltham, MA, USA) after denaturalization and annealing. As the BV-173 cell line contains the IKaros6 transcript, this cell line was employed as the positive control for these experiments (DMSZ, Braunschweig, Germany).

Reverse transcription polymerase chain reaction (RT-PCR). RT was performed by use of PrimeScript RT-PCR kit (Takara Bio, Inc., Otsu, Japan). Fusion gene fragments were amplified by nested PCR and the primer sets and cycling conditions were designed as Niels Pallisgaard's publication (7) (Table I). Fifteen microliters of PCR product was added in a $1.5 \%$ agarose gel to electrophorese for $60 \mathrm{~min}$ at $100 \mathrm{~V}$.

Cloning and sequencing. PCR product was purified from the agarose gel mentioned above and inserted in pEASY-T1 Cloning Vector provided by pEASY-T1 Cloning kit (TransGen Biotech Co., Ltd., Beijing, China). Transformation and positive clone detection were performed by recommended protocol for the kit. Plasmid DNA of positive clone was extracted by GenElute Plasmid Miniprep kit (Sigma-Aldrich; Merck KGaA, Darmstadt, Germany) and sequenced on AB 3500 Genetic Analyzer (Applied Biosystems; Thermo Fisher Scientific, Inc.). PCR primers for positive clone detection and sequencing primer were provided in pEASY-T1 Cloning kit.

Probe detection. To monitor the load of BCR/ABL fusion gene, a TaqMan probe was designed on BCR exon 14 (5'-CGT CCACTCAGCCACTGGATTTAAGCA-3'). PCR primer sequences were 5'-GGGCTCTATGGGTTTCTGAATGT-3' forward and 5'-AGACCCGGAGCTTTTCACTTG-3' reverse. RCR ran in StepOnePlus Real-Time PCR system (Applied Biosystems; Thermo Fisher Scientific, Inc.) and condition was default settings.

\section{Results}

The karyotype was 43-47, XY, $+6, \mathrm{t}(9,22)(\mathrm{q} 34 ; \mathrm{q} 11)$, del(9)(p22), + mar, inc [cp5]/46, XY[5]. FISH confirmed the presence of the BCR-ABL translocation with $92 \%$ of cells reporting positive

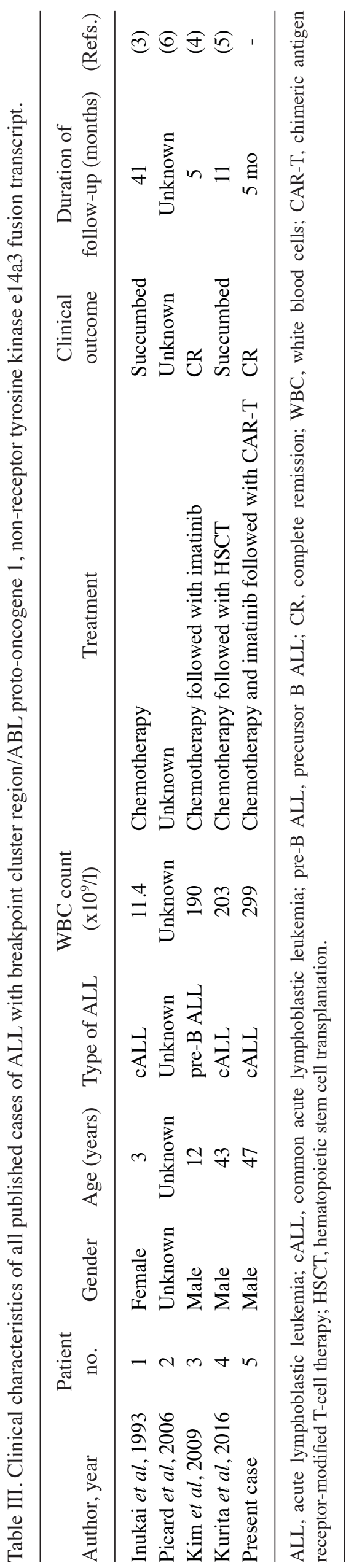


for the translocation with the pattern of 1O1G2F (Fig. 1B). RT-PCR followed with agarose gel electrophoresis showed two abnormal bands approximately 300 bp (Fig. 1C). Cloning and Sanger sequencing revealed a fusion of BCR e14 and ABL a3 without any extra insertion or deletion (Fig. 1D). During the course of disease, BCR/ABL fusion gene probe detection have been done for 5 times which depicted the rise and fall of the $\mathrm{Ph}$ positive cell clones in profile (Table II).

\section{Discussion}

The reason why $\mathrm{BCR} / \mathrm{ABL}$ e14a3 is so rare has not been discussed too much. Considering the intron between al and a2 has a large size of approximately $140 \mathrm{~kb}$ and the intron between a 2 and a 3 has only approximately $0.5 \mathrm{~kb}$, the answer seems to be clear. The breakpoint may randomly locate in any part of the region, and based on the principle of probability, there is a little chance to generate a e14a3 fusion.

Partially due to the limited number of the peculiar cases, the influence of lacking exon a 2 in BCR/ABL fusion to patients' clinical outcome is still controversial (8). More reliable results and conclusions hang on the accumulation of a large number of related cases from laboratories all over the world, and which calls for the extensive use of primer sets that can efficiently detect those rare fusion transcripts. As for ALL, condition gets more complicated considering the heterogeneity of patients and complicated pathogenesis. Kurita et al (5) compared the outcome of ALL patients with BCR/ABL fusions lacking exon $\mathrm{a} 2$ that received imatinib treatment and those who did not, and he found that lacking exon a2 proved to be an adverse factor to prognosis but imatinib therapy brought a good prognosis remarkably. But it's not that every ALL patient with BCR/ABL fusion lacking exon a 2 achieved a complete remission by the additional usage of imatinib, including the one Kurita had reported and the present case.

Most reported ALL cases with e14a3 BCR/ABL fusion transcript had a progressive progression and unfavorable outcome (Table III). Except for BCR/ABL fusion gene, patient of the present case additionally had an IKZF1 deletion which is conformed as a significant adverse prognosis factor (9). After receiving a common chemotherapy, he achieved a cytological remission but soon relapsed. Other than the cases previously published dead without further therapy could be applied, he got a chance and consented to the CAR-T clinical trial, which brought him a complete remission. As a model of new therapies emergent in recent years, CAR-modified T-cell therapy has achieved a series of splendid success. Despite severe associated toxicities occurring occasionally, remarkable therapy effect remains shiny and promises a hopeful future of hematological neoplasm treatment. In theory, CAR-modified $\mathrm{T}$ cells archer and destroy target cells accurately by the identification of cell surface antigens and therefore it's independent of whether the BCR/ABL fusion transcript or drug resistance exists. Patients who do not have a good response with imatinib may have more clinical benefit from CAR-modified T-cell therapy. There is much likelihood that CAR-modified T-cell therapy would be the first-line treatment instead of being the last hope for ALL in the near future and brought an altered choose with TKI for those who carried $\mathrm{Ph}$ chromosome.

\section{References}

1. Faderl S, Talpaz M, Estrov Z, O'Brien S, Kurzrock R and Kantarjian HM: The biology of chronic myeloid leukemia. N Engl J Med 341: 164-172, 1999.

2. Kurzrock R, Shtalrid M, Romero P, Kloetzer WS, Talpas M, Trujillo JM, Blick M, Beran M and Gutterman JU: A novel c-abl protein product in Philadelphia-positive acute lymphoblastic leukaemia. Nature 325: 631-635, 1987.

3. Inukai T, Sugita K, Suzuki T, Ijima K, Goi K, Tezuka T, Kojika S, Hatakeyama K, Kagami K, Mori T, et al: A novel 203 kD aberrant BCR-ABL product in a girl with Philadelphia chromosome positive acute lymphoblastic leukaemia. Br J Haematol 85: 823-825, 1993.

4. Kim J, Park TS, Lyu CJ, Song J, Lee KA, Kim SJ, Lee HJ and Choi JR: BCR/ABL rearrangement with b3a3 fusion transcript in a case of childhood acute lymphoblastic leukemia. Cancer Genet Cytogenet 189: 132-137, 2009.

5. Kurita D, Hatta Y, Hojo A, Kura Y, Sawada U, Kanda Y and Takei M: Adult acute lymphoblastic leukemia with a rare b3a3 type BCR/ABL1 fusion transcript. Cancer Genet 209: 161-165, 2016.

6. Picard C, Hayette S, Bilhou-Nabera C, Cayuela JM, Delabesse E, Frenoy N, Preudhomme C, Dupont M, Bastard C, Bories D, et al: Prospective multicentric molecular study for poor prognosis fusion transcripts at diagnosis in adult B-lineage ALL patients: the LALA 94 experience. Leukemia 20: 2178-2181, 2006.

7. Pallisgaard N, Hokland P, Riishøj DC, Pedersen B and Jørgensen P: Multiplex reverse transcription-polymerase chain reaction for simultaneous screening of 29 translocations and chromosomal aberrations in acute leukemia. Blood 92: 574-588, 1998.

8. Snyder DS, McMahon R, Cohen SR and Slovak ML: Chronic myeloid leukemia with an e13a3 BCR-ABL fusion: Benign course responsive to imatinib with an RT-PCR advisory. Am J Hematol 75: 92-95, 2004.

9. Martinelli G, Iacobucci I, Storlazzi CT, Vignetti M, Paoloni F, Cilloni D, Soverini S, Vitale A, Chiaretti S, Cimino G, et al: IKZF1 (Ikaros) deletions in BCR-ABL1-positive acute lymphoblastic leukemia are associated with short disease-free survival and high rate of cumulative incidence of relapse: A GIMEMA AL WP report. J Clin Oncol 27: 5202-5207, 2009. 